Bull. Austral. Math. Soc.

Vol. 41 (1990) [87-96]

\title{
RELATIVELY CONGRUENCE DISTRIBUTIVE SUBQUASIVARIETIES OF A CONGRUENCE MODULAR VARIETY
}

\author{
Keith A. Kearnes
}

We characterise the relatively congruence distributive subquasivarieties of a modular variety using the modular commutator. Our characterisation allows us to extend the results of Dziobiak concerning relatively congruence distributive quasivarieties of nonassociative $\mathbf{R}$-algebras.

\section{INTRODUCTION}

Don Pigozzi recently extended Baker's finite basis theorem by showing that every finitely generated relatively congruence distributive (RCD) quasivariety is finitely based. Another version of his theorem concerns RCD quasivarieties that need not be finitely generated. However, it is usually very difficult to tell if a class of algebras generates an RCD quasivariety or if a set of quasi-identities define such a quasivariety. Partial results on the characterisation of $\mathrm{RCD}$ quasivarieties were obtained by Czelakowski and Dziobiak in [2]. In particular, they characterised the RCD subquasivarieties of a congruence distributive variety. Later, in [3], Dziobiak obtained a (fairly complicated) characterisation of the RCD subquasivarieties of a congruence permutable variety. The purpose of our paper is to provide a characterisation of all RCD subquasivarieties of a modular variety.

Our choice of terminology and notation for commutator theory and congruences follows [4] and for universal algebra follows [1].

\section{RCD QUASIVARIETIES}

We begin with some definitions.

DEFINITION 2.1: A quasi-identity is a universally quantified first-order sentence of the form:

$$
\bigwedge_{i<n}\left(p_{i}\left(x_{1}, \ldots, x_{k}\right) \approx q_{i}\left(x_{1}, \ldots, x_{k}\right)\right) \Rightarrow p\left(x_{1}, \ldots, x_{k}\right) \approx q\left(x_{1}, \ldots, x_{k}\right) .
$$

Received 7 February 1989

Copyright Clearance Centre, Inc. Serial-fee code: 0004-9729/90 \$A2.00+0.00. 
A quasivariety is a class of algebras defined by quasi-identities. Alternately, a quasivariety is a class of algebras closed under isomorphisms (I), subalgebras (S), products (P) and ultraproducts $\left(\mathbf{P}_{U}\right)$. (The only other class operator that we will mention is the operator $\mathbf{P}$, that closes a class under the formation of subdirect products.)

If $K$ is a quasivariety, $\mathbf{A}$ is of the type of $K$ and $\theta$ is a congruence on $\mathbf{A}$ satisfying $\mathbf{A} / \boldsymbol{\theta} \in K$, then we call $\theta$ a $K$-congruence. The collection of $K$-congruences on $\mathbf{A}$, ordered by inclusion, is a complete lattice which we call the $K$-congruence lat tice of $A$. We denote it by $K$-Con $\mathbf{A}$. We say that $\mathbf{A} \in K$ is relatively congruence distributive (abbreviated RCD) if $K$-Con $\mathbf{A}$ is a distributive lattice. $K$ is RCD if every member is.

We will use a subscript notation to denote certain classes of algebras contained in a quasivariety. The next definition explains what these subscripts mean.

Definition 2.2: An algebra $\mathbf{A}$ in a quasivariety $K$ is relatively subdirectly irreducible (RSI) if $\mathbf{A}$ has a smallest nonzero $K$-congruence. $K_{R S I}$ denotes the class of RSI members of $K . K_{S I}$ denotes the class of (absolutely) subdirectly irreducible members of $K$.

$\mathbf{A}$ is finitely subdirectly irreducible (FSI) if no two nonzero congruences of $\mathbf{A}$ intersect in the zero congruence. $K_{F S I}$ denotes the class of FSI members of $K$. $K_{R F S I}$ denotes the class of relatively finitely subdirectly irreducible algebras, that is, the class of algebras in $K$ for which no two nonzero $K$-congruences intersect the zero congruence.

If $\mathbf{A}$ belongs to a modular variety, then we call $\mathbf{A}$ prime if $[\alpha, \beta]=0$ holds for no pair of nonzero congruences $\alpha$ and $\beta$. Similarly, $\mathbf{A}$ is semiprime if $[\alpha, \alpha]=0$ holds for no nonzero $\alpha$. $K_{\text {prime }}$ denotes the class of prime members of $K$ and $K_{\text {semiprime }}$ denotes the class of semiprime members of $K$. We only use these when $K$ generates a modular variety.

It is quite easy to see that $K_{\text {prime }}=K_{F S I} \cap K_{\text {semiprime }}$.

Now we are ready for our first theorem.

THEOREM 2.3. Let $K$ be a subquasivariety of a congruence modular variety. The following conditions are equivalent:

(a) $K$ is $\mathrm{RCD}$;

(b) $K_{R S I} \subseteq K_{F S I}$ and $K \models_{\text {con }}([\alpha, \alpha] \approx 0 \Rightarrow \alpha \approx 0)$;

(c) $K_{R S I} \subseteq K_{\text {prime }}$.

PROOF: The class of algebras in $K$ satisfying the congruence condition $([\alpha, \alpha] \approx 0 \Rightarrow \alpha \approx 0)$ is closed under IP, so $K \models_{\text {con }}([\alpha, \alpha] \approx 0 \Rightarrow \alpha \approx 0)$ if and only if $K_{R S I} \models_{\text {con }}([\alpha, \alpha] \approx 0 \Rightarrow \alpha \approx 0)$. This shows that condition (b) merely states that $K_{R S I} \subseteq K_{F S I} \cap K_{\text {semiprime }}$. From the observation preceding the statement of the theorem, it follows that $(b)$ and $(c)$ are equivalent. We will now show that $(a)$ and $(b)$ are equivalent. 
Assume that $K$ is RCD. Theorem 2 of [3] proves that $K_{R F S I}=K_{F S I}$ so we have $K_{R S I} \subseteq K_{R F S I}=K_{F S I}$. Now suppose that $\mathrm{A} \in K$ has a congruence $\alpha$ such that $[\alpha, \alpha]=0$. Then let $\mathbf{B}$ be the subalgebra of $\mathbf{A} \times \mathbf{A} \times \mathbf{A}$ consisting of the triples $(x, y, z)$ where $x / \alpha=y / \alpha=z / \alpha$. Since we are in a modular variety we have a ternary difference term $d$ and it defines a mapping $d: \mathbf{B} \rightarrow \mathbf{A}:(x, y, z) \mapsto d(x, y, z)$. By Proposition 5.7 of [4], this mapping is a homomorphism. $A$ is in $K$, so $\delta=k e r d$ is a $K$-congruence. Denote by $\eta_{0}, \eta_{1}$ and $\eta_{2}$ the kernels of the coordinate projections from $\mathbf{B}$ to $\mathbf{A}$. These three congruences are $K$-congruences since they are kernels of homomorphisms from $\mathbf{B}$ to $\mathbf{A} \in K$.

The congruence $\delta \cdot \eta_{0} \cdot \eta_{1}$ consists of pairs $((r, s, t),(r, s, u))$ such that $d(r, s, t)=$ $d(r, s, u)$. Using the $\alpha$-term condition we see that $d(t, t, t)=d(t, t, u)$ or $t=u$. Thus, $\delta \cdot \eta_{0} \cdot \eta_{1}=0=\delta \cdot \eta_{1} \cdot \eta_{2}=\delta \cdot \eta_{0} \cdot \eta_{2}$. On the other hand, $d^{-1}(\alpha)=\eta_{0} \cdot \eta_{1}+\eta_{1} \cdot \eta_{2}+\eta_{0} \cdot \eta_{2}$, so $d^{-1}(0)=\delta \subseteq \eta_{0} \cdot \eta_{1}+\eta_{1} \cdot \eta_{2}+\eta_{0} \cdot \eta_{2}$. Since $K$ is RCD we have:

$$
0=\delta \cdot\left(\eta_{0} \cdot \eta_{1}+_{K} \eta_{1} \cdot \eta_{2}+K \eta_{0} \cdot \eta_{2}\right) \supseteq \delta \cdot\left(\eta_{0} \cdot \eta_{1}+\eta_{1} \cdot \eta_{2}+\eta_{0} \cdot \eta_{2}\right)=\delta .
$$

(We are using + to denote the join in congruence lattices and $+_{K}$ to denote the join in the $K$-congruence lattice.) This forces the conclusion that $\delta=0$. If there exists $(c, d) \in \alpha \backslash 0$, then $((c, c, c),(c, d, d)) \in \delta \backslash 0=\phi$, which is impossible. It follows that $\alpha=0$.

The only thing that remains is to show that $(b)$ implies $(a)$. Let $K$ be a subquasivariety of a modular variety. Assume that $K_{R S I} \subseteq K_{F S I}$ and that $K$ is not RCD. We will argue that $K \nvdash \nvdash_{\text {con }}([\alpha, \alpha] \approx 0 \Rightarrow \alpha \approx 0)$.

Since $K$ is not $\mathrm{RCD}$, there is an algebra $\mathbf{A} \in K$ and congruences $\rho, \sigma, \tau \in K$. Con $\mathbf{A}$ such that $\theta=\rho \cdot\left(\sigma+_{K} \tau\right)>\rho \cdot \sigma+K \rho \cdot \tau=\delta$. Choose a $\psi \in K$-Con $\mathbf{A}$, completely meet-irreducible in $K$-Con $\mathbf{A}$, such that $\delta \leq \psi$ but $\theta \$ \psi$. From $\theta \$ \psi$ it follows that $\rho \npreceq \psi$ and also that either $\sigma \not \psi$ or $\tau \not \psi$ (since $\sigma+\kappa \tau \npreceq \psi$.) We assume that $\sigma \not \psi$.

$\mathbf{A} / \psi \in K_{R S I} \subseteq K_{F S I}$, so $\psi$ is meet-irreducible in Con $\mathbf{A}$. We have $\rho+\psi, \sigma+\psi$ $>\psi$, so $\alpha^{\prime}=(\rho+\psi) \cdot(\sigma+\psi)>\psi$. Notice also that

$$
\left[\alpha^{\prime}, \alpha^{\prime}\right]+\psi \leq[\rho+\psi, \sigma+\psi]+\psi=[\rho, \sigma]+\psi \leq \rho \cdot \sigma+\psi=\psi
$$

That is, $\alpha^{\prime}>\psi$ but $\left[\alpha^{\prime}, \alpha^{\prime}\right] \leq \psi$. Since $\psi$ is a $K$-congruence, $\mathbf{B}=\mathbf{A} / \psi$ is a member of $K$. Setting $\alpha=\alpha^{\prime} / \psi$, we see that $\alpha$ is a nonzero congruence on $\mathbf{B}$ satisfying $[\alpha, \alpha]$ $=0$. Hence, $K \forall \forall_{\text {con }}([\alpha, \alpha] \approx 0 \Rightarrow \alpha \approx 0)$. This finishes the proof of this theorem.

Corollary 2.4. Let $K$ be a subquasivariety of a congruence distributive variety. $K$ is $\mathrm{RCD}$ if and only if $K_{R S I} \subseteq K_{F S I}$.

Compare this corollary to the characterisation of RCD subquasivarieties of $C D$ varieties given by $\mathrm{Czelakowski}$ and Dziobiak. Their version states that $K$ is $\mathrm{RCD}$ if 
and only if $K_{R F S I} \subseteq K_{F S I}$. Our version, which requires only that $K_{R S I} \subseteq K_{F S I}$, is a slightly stronger statement since $K_{R S I} \subseteq K_{R F S I}$.

For the next corollary we will denote the monolith of a subdirectly irreducible algebra $\mathbf{A}$ by $\mu_{A}$.

Corollary 2.5. Let $K=\operatorname{ISPP}_{U}(M)=\operatorname{ISP}(M)$ where $M$ is a finite set of finite algebras contained in some congruence modular variety. $K$ is RCD if and only if $K_{R S I}=K_{S I}$ and for each $\mathbf{A} \in K_{S I}$ we have $\left[\mu_{A}, \mu_{A}\right]=\mu_{A}$.

Note that in this situation one always has $K_{S I} \subseteq K_{R S I} \subseteq \mathbf{S}(M)$.

The condition $\left[\mu_{\mathrm{A}}, \mu_{\mathrm{A}}\right]=\mu_{\mathrm{A}}$ for $\mathbf{A} \in K_{S I}$ says precisely that $K_{S I} F_{\text {con }}$ $([\alpha, \alpha] \approx 0 \Rightarrow \alpha \approx 0)$. $K_{F S I}=K_{S I}$ since both classes contain only finite algebras. Thus, the conditions of the corollary hold if and only if $K_{R S I}=K_{S I}=K_{\text {prime }}$. Therefore, this corollary follows from part (c) of the theorem.

The corollary shows that it is not hard to apply Theorem 2.3 in the case that $K$ is generated by a finite set $M$ of finite algebras. One only has to examine the homomorphisms between the algebras in $\mathbf{S}(M)$ to determine the members of $K_{R S I}$. At this point, checking the conditions in the theorem is a routine investigation of the congruences of the algebras in $K_{R S I}$. On the other hand, it is usually not easy to verify the condition 2.3(b) for an arbitrary quasivariety. Indeed, it is not even clear where to start looking for quasivarieties for which $2.3(\mathrm{~b})$ or (c) holds. For this reason, we devote the next section to describing a way of constructing certain RCD subquasivarieties of modular varieties.

\section{SOME RCD QUASIVARIETIES}

\subsection{The general case.}

Suppose that $P$ is a property of algebras that is preserved by isomorphisms and by the formation of subdirect products and that $\mathcal{W}$ is a variety or quasivariety. Say that a quasivariety has $\mathrm{P}$ if all its members do. Now, if $L$ is a subquasivariety of $W$ that has $\mathrm{P}$, then any quasivariety $L^{\prime} \subseteq L$ also has $\mathrm{P}$. If $L$ and $K$ are subquasivarieties of $\mathcal{W}$ that have $\mathrm{P}$, then $K \vee L=\operatorname{IP} \triangleleft(K \cup L)$ has $\mathrm{P}$. Thus, the subquasivarieties of $\mathcal{W}$ that have $\mathbf{P}$ form a lattice ideal in the lattice of subquasivarieties of $\mathcal{W}$. If $\mathcal{W}$ is locally finite, then $\mathcal{W}$ contains a largest subquasivariety that has $P$.

Let us examine the congruence condition $\forall \alpha([\alpha, \alpha] \approx 0 \Rightarrow \alpha \approx 0)$ which defines the class of semiprime algebras. The class of semiprime algebras in a congruence modular variety is closed under IP.. By our general arguments, every locally finite, congruence modular variety has a largest semiprime subquasivariety. In fact, we will show that a congruence modular variety satisfying a much weaker finiteness hypothesis always has a largest semiprime subquasivariety, and that this quasivariety can be axiomatised by a 
single quasi-identity (which we show how to construct). This will give us a good place to start looking for RCD quasivarieties, since any RCD subquasivariety of a congruence modular variety consists of semiprime algebras.

THEOREM 3.1. Let $\mathcal{V}$ be a congruence modular variety and assume that $K$ is a subquasivariety of $\mathcal{V}$. Suppose that on $\mathbf{F}_{\mathcal{V}}(u, v)$ the congruence $[\Theta(u, v), \Theta(u, v)]$ is finitely generated, say that $[\Theta(u, v), \Theta(u, v)]=\Theta\left(\left(p_{0}(u, v), q_{0}(u, v)\right), \ldots\right.$, $\left.\left(p_{n-1}(u, v), q_{n-1}(u, v)\right)\right)$. Then the following conditions are equivalent:

(a) $K \models_{\text {con }}([\alpha, \alpha] \approx 0 \Rightarrow \alpha \approx 0)$.

(b) $K \vDash\left(\bigwedge_{i<n}\left(p_{i}(x, y) \approx q_{i}(x, y)\right) \Rightarrow x \approx y\right)$.

(Here and elsewhere we abuse notation by using the symbols $p_{i}$ and $q_{i}$ to denote elements of $\mathbf{F}_{\mathcal{V}}(u, v)$ and also to denote some choice of binary terms that represent these elements.)

Proof: Suppose that $(b)$ fails. Then there is an $\mathbf{A} \in K$ containing elements $c \neq d$ such that for all $i<n, p_{i}(c, d)=q_{i}(c, d)$. Now let $\mathbf{A}^{\prime} \in K$ be the subalgebra of $\mathbf{A}$ that is generated by $c$ and $d$. Since $\mathbf{A}^{\prime}$ is 2-generated, $[\Theta(c, d), \Theta(c, d)]$ $=\Theta\left(\left(p_{0}(c, d), q_{0}(c, d)\right), \ldots,\left(p_{n-1}(c, d), q_{n-1}(c, d)\right)\right)=0$ on $\mathbf{A}^{\prime}$, yet $\Theta(c, d) \neq 0$. This witnesses a failure of $(a)$.

Conversely, assume that (a) fails. Then there is a $\mathrm{B}$ in $K$ with a non- zero congruence $\beta$ such that $[\beta, \beta]=0$. Choose $(e, f) \in \beta \backslash 0$ and let $\mathbf{B}^{\prime} \in K$ be the subalgebra of B generated by $e$ and $f$. Of course, $\Theta\left(\left(p_{0}(e, f), q_{0}(e, f)\right), \ldots,\left(p_{n-1}(e, f), q_{n-1}(e, f)\right)\right)$ $\left.\subseteq[\Theta(e, f), \Theta(e, f)] \subseteq[\beta, \beta]\right|_{B}=0$, so for all $i<n, p_{i}(e, f)=q_{i}(e, f)$. Since $e \neq f$, (b) fails.

We should remark that to make this theorem work we only needed to assume that $[\Theta(u, v), \Theta(u, v)]$ generates a finitely generated $K$-congruence of $\mathbf{F}_{\mathcal{V}}(u, v)$. Even if this does not hold, the assumption that $[\Theta(u, v), \Theta(u, v)]$ is finitely generated is only needed to prove that $(a)$ implies $(b)$. If $[\Theta(u, v), \Theta(u, v)]$ is not finitely generated, then we can still choose $\left\{\left(p_{i}, q_{i}\right) \mid i<n\right\}$ to be any finite subset of $[\Theta(u, v), \Theta(u, v)]$ and construct a quasi-identity as in part $(b)$ which defines a quasivariety of semiprime algebras. Of course, it need not be the largest quasivariety of semiprime algebras, but it does give us a place to start looking for RCD quasivarieties.

We eventually want to apply this result to quasivarieties of nonassociative $\mathbf{R}$ algebras over a commutative ring $\mathbf{R}$. For this application we prefer a slightly different statement of the theorem.

We say that a variety $\mathcal{V}$ is 0 -regular, or consists of 0 -regular algebras, if $\mathcal{V}$ has an equationally definable constant denoted 0 and $\mathcal{V}$ has the property that any congruence $\boldsymbol{\theta}$ on an algebra $\mathbf{A} \in \mathcal{V}$ is determined by the $\theta$-class $0 / \theta$. For example, the varieties of groups, rings or modules are 0 -regular. Hagemann has shown in [5] that 0 -regular vari- 
eties are congruence modular and n-permutable for some $n$. The variety of implication algebras is an example of a 0 -regular variety that is 3 -permutable but not permutable. We leave the proof of the following version of Theorem 3.1 to the reader.

THEOREM 3.2. Let $\mathcal{V}$ be 0 -regular and $K$ be a subquasivariety of $\mathcal{V}$. Suppose that on $\mathrm{F}_{\mathcal{V}}(u)$ the congruence $[\Theta(u, 0), \Theta(u, 0)]$ is finitely generated, say that $[\Theta(u, 0), \Theta(u, 0)]=\Theta\left(\left(t_{0}(u), 0\right), \ldots,\left(t_{n-1}(u), 0\right)\right)$. Then the following conditions are equivalent:
(a) $K \models_{\text {con }}([\alpha, \alpha] \approx 0 \Rightarrow \alpha \approx 0)$;
(b) $K \vDash\left(\bigwedge_{i<n}\left(t_{i}(x) \approx 0\right) \Rightarrow x \approx 0\right)$.

Recalling Theorem 2.3(b), we see that it would be desirable to have some conditions on a quasivariety $K$ that ensure that $K_{R S I} \subseteq K_{F S I}$ or, better yet, conditions insuring that $K_{R S I} \subseteq K_{\text {prime }}$. For this we need more notation.

Let $\mathbf{F}=\mathbf{F}_{\mathcal{V}}(r, s, u, v)$ (or $\mathbf{F}_{\mathcal{V}}(u, v)$ if $\mathcal{V}$ is 0 -regular). Let $\mathrm{S}$ be a finite subset of pairs

$$
S=\left\{\left(p_{i}(r, s, u, v), q_{i}(r, s, u, v)\right) \in\left[\Theta_{F}(r, s), \Theta_{F}(u, v)\right] \mid i<n\right\}
$$

or

$$
S=\left\{\left(t_{i}(u, v), 0\right) \in\left[\Theta_{F}(u, 0), \Theta_{F}(v, 0)\right] \mid i<n\right\}
$$

if $\mathcal{V}$ is 0 -regular). Define $\varphi_{s}$ to be the universal sentence

$$
\begin{gathered}
\forall x, y, z, w\left(\bigwedge_{i<n}\left(p_{i}(x, y, z, w) \approx q_{i}(x, y, z, w)\right) \Rightarrow x \approx y \text { or } z \approx w\right) \\
\forall x, y\left(\bigwedge_{i<n}\left(t_{i}(x, y) \approx 0\right) \Rightarrow x \approx 0 \text { or } y \approx 0\right)
\end{gathered}
$$

if $\mathcal{V}$ is 0 -regular). Let $M_{\varphi}$ denote the class of algebras in $\mathcal{V}$ satisfying the sentence $\varphi$.

ThEOREM 3.3. Let $\mathcal{V}$ be congruence modular and let $S$ and $M_{\varphi}$ be defined as in the previous paragraph where $\varphi=\varphi_{S}$. The following are true:

(a) $M_{\varphi}$ consists of prime algebras;

(b) $K=\operatorname{ISPP}_{U}\left(M_{\varphi}\right)$ is $\mathrm{RCD}_{\text {; }}$

(c) $K_{R S I} \subseteq M_{\varphi}=K_{\text {prime }}$;

(d) $L$ is an RCD subquasivariety of $K$ if and only if $L=\operatorname{ISPP}_{U}(N)$ for some class $N$ satisfying $\varphi$.

Proof: We argue the general version and leave to the reader the proof of the refined version of this theorem that holds when $\mathcal{V}$ is 0 -regular. 
Suppose that $(a)$ is false. Then $M_{\varphi}$ contains an algebra $\mathbf{A}$ which has nonzero congruences $\alpha$ and $\beta$ such that $[\alpha, \beta]=0$. Choose $(e, f) \in \alpha \backslash 0$ and $(g, h) \in \beta \backslash 0$. Let $\mathbf{A}^{\prime}$ be the subalgebra of $\mathbf{A}$ that is generated by $e, f, g$ and $h$. Since $M_{\varphi}$ is defined by a universal sentence, $\mathbf{A}^{\prime} \in M_{\varphi}$. Also, $\left.[\Theta(e, f), \Theta(g, h)] \subseteq[\alpha, \beta]\right|_{A^{\prime}}=0$. Therefore, by the way that $\mathrm{S}$ was chosen, for all $i<n$ we have $p_{i}(e, f, g, h)=q_{i}(e, f, g, h)$. But $e \neq f$ and $g \neq h$. Thus, $\mathbf{A}^{\prime} \not \neq \varphi$, which is a contradiction to the fact that $\mathbf{A}^{\prime} \in M_{\varphi}$.

For part $(b), K=\operatorname{ISPP}_{U}\left(M_{\varphi}\right)=\operatorname{IP}_{s} \operatorname{SP}_{U}\left(M_{\varphi}\right)$. Since $M_{\varphi}$. is a universal class, $\operatorname{ISP}_{U}\left(M_{\varphi}\right)=M_{\varphi}$. Hence, $K=\mathbf{I P}_{s}\left(M_{\varphi}\right)$. It follows that $K_{R S I} \subseteq M_{\varphi}$ and so, by part (a) of this theorem, $K_{R S I} \subseteq K_{\text {prime }}$. This is condition (c) of Theorem 2.3. It follows that $K$ is $\mathrm{RCD}$.

To prove part (c), we need to show that $K_{\text {prime }} \subseteq M_{\varphi}$. The other inclusions $K_{R S I}$ $\subseteq M_{\varphi} \subseteq K_{\text {prime }}$ follow from part $(a)$ and part $(b)$. We assume that $\mathbf{A} \in K_{\text {prime }} \backslash M_{\varphi}$ and will argue to a contradiction. Since $K=\operatorname{IP}\left(M_{\varphi}\right)$, we may assume that $\mathbf{A}$ is subdirectly embedded into the product $\Pi_{i \in I} \mathbf{A}_{i}$ where $\mathbf{A}_{i} \in M_{\varphi}$. Recall that $\varphi$ is the sentence

$$
\forall x, y, z, w\left(\bigwedge_{j<n}\left(p_{j}(x, y, z, w) \approx q_{j}(x, y, z, w)\right) \Rightarrow x \approx y \text { or } z \approx w\right) .
$$

Since $\mathbf{A}$ fails $\varphi\left(\mathbf{A} \notin M_{\varphi}\right)$, we can find elements of $\mathbf{A}, a \neq b$ and $c \neq d$, such that for all $j$ we have $p_{j}(a, b, c, d)=q_{j}(a, b, c, d)$. Of course, in the $i^{\text {th }}$ coordinate we have $p_{j}\left(a_{i}, b_{i}, c_{i}, d_{i}\right)=q_{j}\left(a_{i}, b_{i}, c_{i}, d_{i}\right)$ for all $j<n$ so, since $\mathbf{A}_{i} \models \varphi$, it must be that $a_{i}=$ $b_{i}$ or $c_{i}=d_{i}$ holds for each $i \in I$. Let us introduce the notation $\llbracket x=y \rrbracket$ for elements $\boldsymbol{x}$ and $y$ of $\Pi_{i \in I} \mathbf{A}_{i} . \llbracket x=y \rrbracket$ denotes the subset of those $i \in I$ for which $x_{i}=y_{i}$. Our observation that for each $i \in I$ either $a_{i}=b_{i}$ or $c_{i}=d_{i}$ can be expressed as $[a=b \rrbracket \cup \llbracket c=d \rrbracket=I$. Let $\alpha$ be the congruence on $\mathbf{A}$ that is the kernel of the natural projection of $\mathbf{A}$ into $\Pi_{i \in \llbracket a=b l} \mathbf{A}_{i}$ and let $\beta$ be the kernel of the projection into $\Pi_{i \in[c=d]} \mathbf{A}_{i}$. Since $\llbracket a=b \rrbracket \cup \llbracket c=d \rrbracket=I$, we get $\alpha \cdot \beta=0$. However, $\alpha \supseteq \Theta(a, b)>$ 0 and $\beta \supseteq \Theta(c, d)>0$. Thus $\mathbf{A}$ is not FSI. This contradicts our assumption that $\mathbf{A}$ $\in K_{\text {prime }} \subseteq K_{F S I}$.

For $(d)$, suppose that $L$ is RCD. $L=\operatorname{ISPP}_{U}\left(L_{R S I}\right)$, so let $N=L_{R S I}$. We will be done with the "only if" statement if we show that $N \models \varphi$; equivalently, that $N \subseteq M_{\varphi}$. Using Theorem 2.3(c), the fact that $L$ is RCD and part (c) of this theorem, we see that

$$
N=L_{R S I} \subseteq L_{\text {prime }} \subseteq K_{\text {prime }}=M_{\varphi}
$$

which finishes this direction. For the other direction, assume that $N$ satisfies $\varphi$ and that $L=\operatorname{ISPP}_{U}(N)$. Then $N \subseteq M_{\varphi}$ so $L \subseteq K$. Further, $L=\operatorname{IP}_{\triangleleft} \operatorname{SP}_{U}(N)$, so $L_{R S I} \subseteq \operatorname{ISP}_{U}(N) \subseteq M_{\varphi} \cap L \subseteq L_{\text {prime }}$. This implies that $L$ is RCD by part (c) of 2.3 . D 
This theorem gives us a concrete way of producing an RCD subquasivariety of a congruence modular variety. Further, it gives a simple characterisation of all the RCD quasivarieties contained in that first one. Our next subsection contains applications of this result.

\subsection{QUASIVARIETIES OF R-ALGEBRAS}

In [3], Dziobiak uses his characterisation of RCD subquasivarieties of permutable varieties to investigate quasivarieties of nearly-associative algebras over a commutative ring $\mathbf{R}$. We use our results to show how to extend his.

Everything in this subsection takes place within the variety $\mathcal{R}$ of (not necessarily associative) $\mathbf{R}$-algebras. This variety is, of course, congruence permutable and 0 -regular. Every congruence on an R-algebra corresponds naturally with an ideal: the ideal associated with a congruence is just the congruence class containing 0 . The commutator in this variety, expressed in terms of ideals, is computed the following way: if $I$ and $J$ are ideals, then $[I, J]=I \cdot J+J \cdot I$. We leave it to the reader to verify this claim; we suggest mimicing the arguments in Chapter 1 of [4].

Theorem 3.2 applies to the variety of $\mathbf{R}$-algebras. In $\mathbf{F}_{\mathcal{R}}(u)$ the congruence $\Theta(u, 0)$ corresponds to the ideal $(u)$. We have $[(u),(u)]=(u) \cdot(u)$. It is not true that $(a) \cdot(b)$ $=(a \cdot b)$ for an arbitrary pair of principal ideals in an arbitrary nonassociative algebra, but it is true (and easy to see) that $(u) \cdot(u)=(u \cdot u)$ in $\mathbf{F}_{\mathcal{R}}(u)$. Hence, let $S=$ $\{(t(u), 0)\}$ where $t(x)=x \cdot x$. The conclusion of Theorem 3.2 is that the quasivariety axiomatised by $(x \cdot x \approx 0 \Rightarrow x \approx 0)$ is the largest quasivariety of semiprime $\mathbf{R}$-algebras. Call this quasivariety $N$. Every RCD subquasivariety of $\mathcal{R}$ is contained in $N . N$ is not RCD itself as the following example shows.

EXAMPLE 3.4. Let $V$ be the 3-dimensional vector space over the 2-element field $\mathbf{F}_{2}$ that is spanned by the vectors $u, v$ and $w$. Define a multiplication on the basis vectors as follows:

\begin{tabular}{|c||c|c|c|}
\hline & $u$ & $v$ & $w$ \\
\hline \hline$u$ & $v$ & 0 & $w$ \\
\hline$v$ & $v$ & $v$ & 0 \\
\hline$w$ & $w$ & 0 & $w$ \\
\hline
\end{tabular}

Extend this multiplication to $V$ by linearity and call the resulting $\mathbf{F}_{2}$-algebra $\mathbf{A}$. If $x=r u+s v+t w$, where $r, s, t \in \mathbf{F}_{2}$, and $(r u+s v+t w)^{2}=(r+s+r s) v+t w=0$, then $r=s=t=0$. Hence, $\mathbf{A} \models\left(x^{2} \approx 0 \Rightarrow x \approx 0\right)$ and so $\mathbf{A} \in N$. A $\notin N_{F S I}$ since $(v) \cap(w)=(0)$. However, $\mathbf{A} \in N_{R S I}$. This is because any ideal $I \subseteq \mathbf{A}$ with $\boldsymbol{w} \notin$ $I$ has $v \in I$. Further, the $N$-ideal generated by $v$ contains $w$. Thus, any nonzero $N$-ideal contains $w$, implying that $\mathbf{A}$ is subdirectly irreducible relative to $N$. Since $\mathbf{A}$ 
$\in N_{R S I} \backslash N_{F S I}, N$ is not RCD.

Although $N$ is not RCD, it seems to be close to being RCD. For example, the associative algebras in $N$ do constitute an RCD subquasivariety, as we shall see.

Let's apply Theorem 3.3 to locate some RCD subquasivarieties of $\mathcal{R}$. First, let $S$ $=\{(u v, 0)\} \subseteq[(u),(v)]=(u)(v)+(v)(u)$. Then $\varphi s$ is the sentence

$$
\forall x, y(x y \approx 0 \Rightarrow x \approx 0 \text { or } y \approx 0) \text {. }
$$

$M_{\varphi}$ is precisely the class of $\mathrm{R}$-algebras without nonzero zero-divisors. From 3.3 we get:

COROLlaRY 3.4. Let $K$ be the quasivariety of $\mathbf{R}$-algebras generated by all $\mathbf{R}$ algebras without nonzero zero-divisors. $K$ is RCD and a subquasivariety $L \subseteq K$ is RCD if and only if $L=\operatorname{ISPP}_{U}(N)$ where $N$ consists of R-algebras without nonzero zero-divisors.

This result was first proved by Dziobiak in [3]. A result of Rjabuhin in [6] is that the quasivariety generated by all $\mathbf{R}$-algebras without nonzero zero-divisors can be axiomatised by $\left(x^{2} \approx 0 \Rightarrow x \approx 0\right)$ and $(x(y z) \approx 0 \Leftrightarrow(x y) z \approx 0)$. It follows that the quasivariety of associative $\mathrm{R}$-algebras satisfying $\left(x^{2} \approx 0 \Rightarrow x \approx 0\right)$ is $\mathrm{RCD}$ and that this is the largest RCD quasivariety of associative $\mathbf{R}$-algebras.

We have the machinery to extend Dziobiak's result. We merely have to go back to Theorem 3.3 and choose a larger subset $\bar{S}$. Say $\bar{S}=\{(u v, 0),(v u, 0)\} \subseteq(u)(v)+(v)(u)$. Then $\varphi \bar{S}$ is:

$$
\forall x, y((x y \approx 0) \&(y x \approx 0) \Rightarrow x \approx 0 \text { or } y \approx 0)
$$

Corolla RY 3.5. Let $\bar{K}$ be the quasivariety of $\mathbf{R}$-algebras generated by all $\mathbf{R}$ algebras satisfying (3). $\bar{K}$ is RCD and a subquasivariety $\bar{L} \subseteq \bar{K}$ is RCD if and only if $\bar{L}=\operatorname{ISPP}_{U}(\bar{N})$ where $\bar{N}$ consists of R-algebras satisfying (3).

We give an example to show that the quasivariety $\bar{K}$ from the last corollary properly contains the quasivariety $K$ from the corollary preceding it.

EXAMPLE 3.5. Of course, $K \subseteq \bar{K}$. Now let $W$ be the 2-dimensional vector space over $\mathbf{F}_{2}$ spanned by $u$ and $v$. Define a bilinear multiplication on $W$ determined by the values:

\begin{tabular}{|l||l|l|}
\hline & $u$ & $v$ \\
\hline \hline$u$ & $v$ & 0 \\
\hline$v$ & $v$ & $v$ \\
\hline
\end{tabular}

Call the resulting $\mathbf{F}_{2}$-algebra $\mathbf{B}$. In $\mathbf{B}$, the only nontrivial products that equal 0 are $u v$, $(u+v) u$ and $v(u+v)$. Hence, $\mathrm{B} \models((x y \approx 0) \&(y x \approx 0) \Rightarrow x \approx 0$ or $y \approx 0)$. However, 
$\mathbf{B} \not \models(x y \approx 0 \Rightarrow x \approx 0$ or $y \approx 0)$. Thus, $\mathbf{B} \in \bar{K}_{\text {prime }} \backslash K_{\text {prime }}$, and it follows that $\mathbf{B}$ $\in \bar{K} \backslash K$.

If we alter the multiplication in $B$ so that on the basis vectors multiplication is given by:

\begin{tabular}{|l||l|l|}
\hline & $u$ & $v$ \\
\hline \hline$u$ & $v$ & 0 \\
\hline$v$ & 0 & $u$ \\
\hline
\end{tabular}

we get an algebra which we call $\mathbf{C}$. $\mathbf{C} \notin \bar{K}$, but $\mathbf{C}$ satisfies

$$
\forall x, y((x y \approx 0) \&(y x \approx 0) \&((x x) y \approx 0) \Rightarrow x \approx 0 \text { or } y \approx 0) .
$$

This sentence is just $\varphi_{\overline{\bar{S}}}$ for $\overline{\bar{S}}=\{(u v, 0),(v u, 0),((u u) v, 0)\} \subseteq(u)(v)+(v)(u)$. The quasivariety generated by the $\mathbf{R}$-algebras satisfying (4) is RCD and it properly contains $\bar{K}$. This leaves us with questions.

Problem 1. Is there a largest RCD quasivariety of $\mathbf{R}$-algebras? Does the answer depend on $\mathbf{R}$ ?

We guess that the answer to both of our questions is probably "no". It is not too hard to show that if $\mathbf{R}=\mathbf{Z L}=$ the ring of integers, then no choice of $S$ in Theorem 3.3 yields a largest RCD quasivariety of $\mathrm{Z}$-algebras. That is, for any finite subset $S \subseteq[(u),(v)]$ there is a $\mathbf{Z}$-algebra $\mathrm{X}$ and a finite subset $S^{\prime}$ such that $S \subseteq S^{\prime} \subseteq[(u),(v)]$ and $\mathbf{X} \vDash \varphi_{S^{\prime}}$ but $\mathbf{X} \not \varphi_{S}$. Of course, this does not solve the problem even for $\mathbf{R}$ $=\mathbf{Z}$, since Theorem 3.3 always produces RCD quasivarieties $K$ in which $K_{\text {prime }}$ is a universal class and there may be other RCD quasivarieties.

\section{REFERENCES}

[1] S. Burris and H. P. Sankappanavar, 'A Course in Universal Algebra', in Graduate Texts in Mathematics 78 (Springer-Verlag, New York, 1981).

[2] J. Czelakowski and W. Dziobiak, 'Quasivarieties with EDPI. A supplement to a result of W. J. Blok and D. Pigozzi', (manuscript).

[3] W. Dziobiak, 'Relatively congruence distributivity within quasivarieties of nearly associative $\phi$-algebras', (preprint).

[4] R. Freese and R. McKenzie, 'Commutator Theory for Congruence Modular Varieties', in LMS Lecture Note Series 125. Cambridge (1987).

[5] J. Hagemann, 'On regular and weakly regular congruences', Preprint No. 75, TH Darmstadt.

[6] J. Rjabuhin, 'Algebras without nilpotent elements I, II', Russian, Algebra i Logika 8 (1969). 181-214, 215-240. 